\title{
First-Principles Investigations of Magnetic
}

\section{Anisotropy and Spin-Crossover Behavior of Fe(III)-TBP Complexes}

\author{
Rishu Khurana, ${ }^{+, \ddagger}$ Sameer Gupta, ${ }^{+, \ddagger}$ and Md. Ehesan Ali ${ }^{*}+$ \\ tInstitute of Nano Science and Technology, Phase 10, Sector-64, Mohali, Punjab, 160062, India \\ $\ddagger$ Contributed equally to this work \\ E-mail: ehesan.ali@inst.ac.in
}

\begin{abstract}
With the ongoing efforts to obtain mononuclear 3d-transition metal complexes that manifest slow relaxation of magnetization and hence, can behave as single molecule magnets (SMMs), we have modelled 14 novel Fe(III) complexes out of which nine behave as potential SMMs. These complexes possess large zero-field splitting (ZFS) parameter $D$ in the range of -40 to $-60 \mathrm{~cm}^{-1}$. The first-principles investigation of the ground-spin state applying density functional theory (DFT) and wave-function based multi-configurations methods e.g. SA-CASSCF/NEVPT2 are found to be quite consistent except for few delicate cases with near degenerate spin-states. In such cases, the hybrid B3LYP functional is found to be biased towards high-spin (HS) state. Altering the percentage of exact exchange admixed in B3LYP functional leads to intermediatespin (IS) ground state consistent with the multireference calculations. The origin of large zero field splitting (ZFS) in the Fe(III)-based trigonal bipyramidal (TBP) complexes is investigated and the $D$-values are further tuned by varying the axial ligands with group XV elements (N, P and As) and equatorial halide ligands from $\mathrm{F}, \mathrm{Cl}, \mathrm{Br}$ and
\end{abstract}


I. Furthermore, a number of complexes are identified with very small $\Delta G_{H S-I S}^{\text {adia. }}$ values indicating the possible spin-crossover phenomenon between the bi-stable spin-states.

\section{Introduction}

A number of lanthanide and transition-metal complexes have been observed to retain their magnetization aligned along the magnetic easy axis for a longer period of time after the applied magnetic field is removed. Such class of magnetic molecules is known as single-molecule magnets (SMMs). The SMMs with a single paramagnetic ion are known as single ion magnets (SIMs). Current interest of such SMMs that exhibit a barrier to the magnetic relaxation lies in their prospective applications in the high-density information storage, ${ }^{1}$ quantum computing, ${ }^{2}$ molecular spintronics ${ }^{3,4}$ and magnetic refrigeration. ${ }^{5}$ The slow magnetic relaxation in these molecules is characterized by an energy barrier, $\mathrm{U}_{\text {eff, }}$ to magnetic moment reversal. The barrier to magnetic relaxation is determined by the magnitude of total spin at the ground state as well as the magnitude of the axial anisotropy. ${ }^{6}$ However, it is the axial magnetic anisotropy that primarily pins the magnetic moment of the ground state along the magnetic easy axis. ${ }^{7-9}$ Magnetic anisotropy in SMMs is a collective effect of spin-orbit coupling (SOC) and lower than octahedral symmetry of molecule. ${ }^{10}$

The magnetic properties of single molecule magnets are controlled by the magnitude of magnetic anisotropy, which can be modulated based on rational design of ligands. Mononuclear metal complexes containing rare earth (4f-metal) elements exhibit quite large magnetic anisotropy. ${ }^{11-18}$ The strong spin-orbit coupling and large unquenched orbital contribution to the magnetic moments in 4 f-elements are mostly responsible for the SMM behavior of lanthanides complexes. ${ }^{19}$ In parallel development with lanthanides based SMMs, highly symmetric 3d transition metal complexes with relatively smaller magnetic moments are also gaining popularity in the recent years. Mononuclear $3 \mathrm{~d}$ tran- 
sition metal complexes with high axial symmetry have shown sign of unquenched orbital momentum with considerable spin-orbit coupling and moderate magnetic anisotropy. ${ }^{20-22}$ Also, in some $3 \mathrm{~d}-4 \mathrm{f}$ clusters, slow relaxation has been observed at temperatures comparable to those for 3d-only SMMs. ${ }^{23,24}$

The phenomenon of spin-crossover (SCO) is one of the most apparent examples of molecular bistability in transition metal complexes. It is generally observed in paramagnetic centers having electronic configuration of $3 d^{4}$ to $3 d^{7}$. The spin-state of such complexes can change from ground-spin state to energetically low lying excited spin states (e.g. low-spin (LS), intermediate-spin (IS) and high-spin (HS)). This switching could be brought by application of external stimuli such as magnetic field, redox reaction, pressure or photo irradiation. ${ }^{25-28}$ Spin-crossover is observed in situation where the ligand field splitting is comparable to spin pairing energy. At the molecular scale, the driving force of the spin conversion is the entropy variation due to the metal-ligand bond length changes. ${ }^{29}$ The change in molecular spin states is accompanied by the change in magnetic as well as structural properties. Observation of spin-crossover phenomenon along with the slow magnetic relaxation at high blocking temperature is highly anticipated for potential use as molecular qubits and logic devices. ${ }^{30}$ This will revolutionize the field of quantum devices for high-density data storage and fast processing of information. Mononuclear 3d transition metal complexes hold promise in this direction with both evident properties of magnetism and spin-crossover at molecular level. ${ }^{31,32}$ Ligand field in 3d-transition metal complexes could play central role in controlling the magnetic anisotropy and SCO behavior of molecule. ${ }^{33,34}$ Thus, understanding ligand field derived modulation of anisotropy in conjunction with SCO property can help in designing of molecules with both properties.

Magnetic anisotropy in mononuclear $3 \mathrm{~d}$ single ion magnets is mainly affected by the molecular symmetry, the ligand field, metal-ligand covalency and the spin-orbit coupling of single metal ion. ${ }^{32,35,36}$ High axial symmetry ensures a relatively unquenched 
orbital angular momentum and high first-order spin orbit coupling. Magnetic anisotropy in such molecules can be tuned to improve SMM properties by changing the electronic configuration of the metal center. This can be achieved by variation of donor atoms in ligands to maximise spin-orbit coupling. ${ }^{37}$ In line with these observations, a giant magnetic anisotropy $(D)$ in the range of -400 to $-535 \mathrm{~cm}^{-1}$ was initially predicted using $a b$ initio methods for the Ni(II) trigonal bipyramidal (TBP) complex by Pavlovic et al. ${ }^{38}$ Interestingly, this predicted molecule was later synthesized by Marriott et al. and the magnetic anisotropy was estimated indirectly, with a lower bound set at $|D| \sim 400 \mathrm{~cm}^{-1}$, which complements well with the theoretically predicted value. ${ }^{39}$ The Ni-complex also showed pressure dependent magnetic anisotropy such that with increasing pressure, the geometry becomes distorted leading to change in the ligand field and a rigorous decrease in magnetic anisotropy. ${ }^{40}$ In another study, a penta coordinated Fe(III) complex was reported by Mossin et al. with the intermediate ground-spin state that exhibits slow magnetic relaxation along with the axial ZFS with $D=-11 \mathrm{~cm}^{-1} \cdot{ }^{41}$ In addition to these complexes, there are several other reports in literature which make it evident that pentacoordinated TBP complexes induce high magnetic anisotropy and are promising candidates for the application of magnetic properties. ${ }^{42-44}$ Hence, the detailed investigation of the factors that affect the magnetic anisotropy in TBP complexes related to ligand-field and magneto-structural correlations are absolute necessities.

Magnetic anisotropy of organometallic based SMMs can be improved by changing the donor properties of the ligand. For a set of $\left[\mathrm{Co}(\mathrm{II})(\mathrm{L})_{2} \mathrm{I}_{2}\right]$ complexes in pseudo tetrahedral symmetry with ligand based on group XV elements (L-quinoline, $\mathrm{PPh}_{3}, \mathrm{AsPh}_{3}$ ), axial anisotropy improves with increasing soft nature of ligands in the order $\mathrm{N}<\mathrm{P}<\mathrm{As}^{45}$ Goswami et al. correlated the ZFS value based on metal ligand $\pi$ charge transfer in a series of octahedral $\mathrm{Cr}$ (III) complexes by varying halide ligands. They found that the $\pi$ donor ligand results in easy plane type anisotropy and $\pi$-acceptor ligand in easy axis anisotropy with enhancement in the ZFS values with increase in metal ligand charge 
transfer (MLCT). ${ }^{46}$ In a recent study on hepta-coordinated Co(II) complexes with axial halide ligands, ZFS values were correlated to the Mayer bond order where increase in easy axis anisotropy is observed with decrease in bond order. ${ }^{47}$

Feng et al. synthesized two penta-coordinated $\mathrm{Fe}(\mathrm{III})$ complexes i.e. [ $\left.\left(\mathrm{PMe}_{3}\right)_{2} \mathrm{FeCl}_{3}\right]$ (here, complex 2) and $\left[\left(\mathrm{PMe}_{2} \mathrm{Ph}\right)_{2} \mathrm{FeCl}_{3}\right]$ having close to ideal TBP geometry. The IS state was observed to be the ground state from DC susceptibility measurements data. The spin-crossover to HS state was captured in complex $\left[\left(\mathrm{PMe}_{2} \mathrm{Ph}\right)_{2} \mathrm{FeCl}_{3}\right] .{ }^{48} \mathrm{~A}$ very high value of ZFS i.e. $D=-50 \mathrm{~cm}^{-1}$ for first and a moderate value of $-17 \mathrm{~cm}^{-1}$ for second complex was observed. The authors claimed that the reported $D$ for first complex is the maximum among all the reported Fe(III) complexes. Later, these two Fe(III) based complexes were theoretically investigated using ab initio calculations by Chowdhury et al. and the magnetic anisotropy was found to be significantly influenced by axial ligands. ${ }^{4}$

From theoretical point of view, for the prediction of spin-crossover and magnetic properties in transition metal complexes, DFT calculations based on hybrid functionals are well known to predict the correct ground spin state of transition metal (TM) complexes but in some cases over-stabilize the HS state. ${ }^{50,51}$ In the benchmark study on spin energetics of TM complexes, Radón et al. reported that NEVPT2 calculations reproduced the energy difference well with an error of $<5 \mathrm{kcal} / \mathrm{mol}^{52}$ Saurabh et al. calculated the ZFS parameters in $\mathrm{Ni}(\mathrm{II})$ based complexes using ab initio SA- CASSCF calculations and suggested that significant enhancement in $D$ values can be achieved by tuning the structural distortion in the coordination environment. ${ }^{53}$

In this work, we have explored the electronic structure and magnetic properties of complex 2, applying $a b$ initio density functional theory (DFT) and wave function based multi-configurational methods such as CASSCF and NEVPT2. A close to ideal TBP geometry, small size, neutral charge and half integral spin ground state makes complex 2 the ideal system to exhibit high magnetic anisotropy with further imposing the question, can we enhance the $D$ value by modulating the ligand environment in the Fe(III) TBP 
complexes? To find an answer, we have modelled 14 novel complexes with the aim to study the effect of ligand environment, ligand-metal covalency and ground-spin state on magnetic anisotropy. To explore ligand environment, the donor atoms from group XV (i.e. N, P and As) in the axial ligands are varied and the effect on the magnetic anisotropy is investigated. Additionally, the influence of halide ligands $\left(\mathrm{F}^{-}, \mathrm{Cl}^{-}, \mathrm{Br}^{-}\right.$and $\left.\mathrm{I}^{-}\right)$at the equatorial position is also studied. A detailed investigation of electronic structure with change in ligand environment is carried out and the origin of the spin-crossover and large zero field splitting is explored.

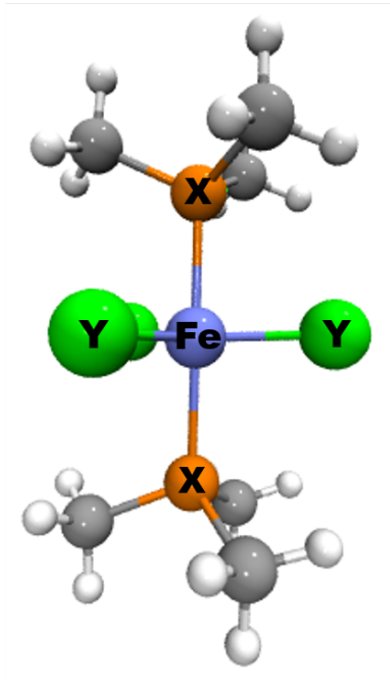

$\begin{array}{cccl}\text { Complex } & \text { X(Axial) } & \text { Y(Equatorial) Acronym } \\ \mathbf{1} & \mathrm{P} & \mathrm{F} & 1_{H S}-\mathrm{PF} \\ \mathbf{2} & \mathrm{P} & \mathrm{Cl} & 2_{I S}-\mathrm{PCl} \\ \mathbf{3} & \mathrm{P} & \mathrm{Br} & 3_{I S}-\mathrm{PBr} \\ \mathbf{4} & \mathrm{P} & \mathrm{I} & 4_{I S}-\mathrm{PI} \\ \mathbf{5} & \mathrm{N} & \mathrm{F} & 5_{H S}-\mathrm{NF} \\ \mathbf{6} & \mathrm{N} & \mathrm{Cl} & 6_{H S}-\mathrm{NCl} \\ \mathbf{7} & \mathrm{N} & \mathrm{Br} & 7_{H S}-\mathrm{NBr} \\ \mathbf{8} & \mathrm{N} & \mathrm{I} & 8_{H S}-\mathrm{NI} \\ \mathbf{9} & \mathrm{As} & \mathrm{F} & 9_{H S}-\mathrm{AsF} \\ \mathbf{1 0} & \mathrm{As} & \mathrm{Cl} & 10_{I S}-\mathrm{AsCl} \\ \mathbf{1 1} & \mathrm{As} & \mathrm{Br} & 11_{I S}-\mathrm{AsBr} \\ \mathbf{1 2} & \mathrm{As} & \mathrm{I} & 12_{I S}-\mathrm{AsI} \\ \mathbf{1 3} & \mathrm{N} / \mathrm{P} & \mathrm{Br} & 13_{I S}-\mathrm{NPBr} \\ \mathbf{1 4} & \mathrm{As} / \mathrm{P} & \mathrm{Br} & 14_{I S}-\mathrm{AsPBr} \\ \mathbf{1 5} & \mathrm{N} / \mathrm{As} & \mathrm{Br} & 15_{I S}-\mathrm{NAsBr}\end{array}$

Figure 1: Structure of modelled Fe (III) complexes, $\left[\mathrm{Fe}\left(\mathrm{XMe}_{3}\right)_{2}(\mathrm{Y})_{3}\right]$. Here, $\mathrm{X}=\mathrm{P}, \mathrm{N}, \mathrm{As}$ and $\mathrm{Y}=\mathrm{F}, \mathrm{Cl}, \mathrm{Br}$, I. The complexes are acronymed based on the serial number followed by ground-spin state (as subscript) and ligands attached to it at axial and equatorial positions respectively.

Complexes 1, 3 and 4 are designed replacing all the equatorial chloride ligand in 2 by fluoride, bromide and iodide respectively. Complexes 5, 6, 7 and 8 contain trimethylamine and complexes $\mathbf{9}, \mathbf{1 0}, \mathbf{1 1}$ and $\mathbf{1 2}$ contain trimethylarsine at the axial positions replacing trimethylphosphine in complexes 1, 2, 3 and 4 respectively. Complex 13 at axial position contains trimethylamine and trimethylphosphine, while complex $\mathbf{1 4}$ contains trimethylphospine and trimethylarsine and complex 15 has trimethylamine and trimethylarsine with bromide at the equatorial position for the last three complexes. 


\section{Theoretical and Computational Methods}

The molecular geometries of all the modelled complexes are optimized applying density functional theory (DFT) using hybrid B3LYP exchange-correlation functional. ${ }^{54,55}$ The localized atom centered valence triple-zeta with two sets of polarization functions, def2-TZVPP, ${ }^{56}$ basis sets are used for all the calculations. The resolution of identity (RI) approximation, as implemented in ORCA, ${ }^{57,58}$ is used to approximate the various integral accuracy to speed up the computations without losing its accuracy. Def2/JK auxiliary basis sets are used along with RI approximation and chain-of-spheres (RIJCOSX) approximation to exact exchange. ${ }^{59,60}$ A reformulated version of Grimme's DFT dispersion correction with Becke-Johnson damping (DFT-D3(BJ)) is included in the geometry optimization. ${ }^{61,62}$ It turned out that $\mathrm{D} 3(\mathrm{BJ})$ produces accurate molecular geometry and is especially crucial to reproduce the experimental axial metal-ligand distances for complex 2. Increased integration grids (Grid 5 in ORCA convention) and tight SCF convergence criteria are used in all the calculations. ${ }^{63-65}$ The atomic charges are obtained using Löwdin population analysis. ${ }^{66}$ The bond order analysis is based on the Mayer bond order. ${ }^{67}$ Single point energies of the DFT optimized structures are calculated using SA-CASSCF/NEVPT2 calculation on top of ground state geometry to look into spincrossover phenomenon. State-average (SA-CASSCF) calculations are employed over the state-specific (SS-CASSCF) because SS-CASSCF calculations are found to stabilize the HS state as ground state in contrast to the experimentally reported IS ground state for complex 2 (Table S4). For closely lying states, multireference SA-CASSCF calculations are advocated to produce improved result as compared to SS-CASSCF configuration interaction calculation for energy difference between spin-states. SA-CASSCF performs a constraint minimization of a weighted sum over energies of multiple states. With the SA approximation, both ground and excited state wave functions are described by state specific configuration interaction (CI) coefficients but use a global single set of orbitals. ${ }^{68}$ The 
similar observation was made by Roemelt et al. that the state specific energies may not be well converged due to missing balance in the appropriateness of a given active space in recovering part of dynamic correlation. ${ }^{69}$

The zero field splitting (ZFS) i.e. the energy difference of electron spin multiplet sublevels, in the absence of an applied magnetic field, is an important component of the spin-hamiltonian for any open-shell system having spin quantum number $S \geq \frac{1}{2}$. In the modelled spin-hamiltonian, this term is included as

$$
\hat{H}_{m o d}=\hat{\mathbf{S}} \cdot \overline{\bar{D}} \cdot \hat{\mathbf{S}}
$$

where $\overline{\bar{D}}$ is the second rank tensor and $\hat{S}$ is the spin-operator of the ground state. The spin-spin (SS) dipolar interactions and spin-orbit coupling (SOC) are dominant contributors to the ZFS. The $\overline{\bar{D}}$ tensor contains the information of the spatial spin distributions in the molecular systems and is directly associated with magnetic anisotropy and the fundamental magnetic property of the associated molecules.

$\overline{\bar{D}}$ is a symmetric traceless tensor and can be diagonalized in a principal axis frame in cartesian coordinate and expressed as

$$
\begin{gathered}
H^{Z F S}=D\left[S_{z}^{2}-\frac{1}{3} S(S+1)\right]+E\left[S_{x}^{2}-S_{y}^{2}\right] \\
D=D_{z z}-1 / 2\left(D_{x x}+D_{y y}\right) ; E=1 / 2\left(D_{x x}-D_{y y}\right)
\end{gathered}
$$

In the principal axis frame, it can be defined by axial $D$ and transverse $E$ components, which are extracted from the diagonal elements of the $\overline{\bar{D}}$ tensor. The axes for the coordinate system that diagonalizes the $\overline{\bar{D}}$ tensor are chosen to fulfill the condition $0 \leq|E / D| \leq 1 / 3$. The sign of the axial ZFS parameter, $D$, is important in determining the nature of the magnetic property associated with the system. Negative value of $D$ and negligible $E$ value are two prerequisite for a material to act as SMM. 
The ZFS parameters arising from spin-orbit coupling of the ground state and excited state are calculated at the level of quasi-degenerate perturbation theory $(\mathrm{QDPT})^{70}$ and are obtained from effective Hamiltonian $\left(\hat{H}_{e f f}\right),{ }^{71}$ where the Breit-Pauli approximation based on spin-orbit mean field (SOMF) Hamiltonian ${ }^{72}$ is used to account for the spinorbit coupling. Here, SOC interaction is expressed as $\hat{H}_{S O C}$ as the perturbation, treated up to second order on spin-free states (SOF) obtained from non-relativistic CI.

$$
\begin{array}{r}
\left\langle\phi_{i}, M_{S}\left|\hat{H}_{e f f}\right| \phi_{j}, M_{S^{\prime}}\right\rangle=\delta_{i j} \delta_{M_{S} M_{S}^{\prime}}\left\langle\phi_{i}, M_{S}\left|\hat{H}_{e l}\right| \phi_{j}, M_{S^{\prime}}\right\rangle+\left\langle\phi_{i}, M_{S}\left|\hat{H}_{S O C}\right| \phi_{j}, M_{S^{\prime}}\right\rangle+ \\
\sum_{\phi_{k}, M_{S_{k}}} \frac{\left\langle\phi_{i}, M_{S}\left|\hat{H}_{S O C}\right| \phi_{k}, M_{S_{k}}\right\rangle\left\langle\phi_{k}, M_{S_{k}}\left|\hat{H}_{S O C}\right| \phi_{j}, M_{S^{\prime}}\right\rangle}{E_{\phi_{j}}-E_{\phi_{k}}}
\end{array}
$$

where $\hat{H}_{e l}$ is the zeroth order Hamiltonian that accounts for the spin-orbit free interactions, $\delta_{i j}$ and $\delta_{M_{S} M_{S^{\prime}}}$ are Kronecker $\delta$ functions, $\phi_{i}$ and $\phi_{j}$ are spin-orbit free states belonging to the model active space, $\phi_{k}$ is a SOF state belonging to the external space, $E_{\phi_{j}}$ and $E_{\phi_{k}}$ are SOF energies of the SOF states $\phi_{j}$ and $\phi_{k}$ respectively.

Single reference spin orbit free (SOF) states from DFT can be used for spin orbit state interaction (SO-SI). But in most cases, they are insufficient for representation of SOC operator as sufficient number of excited SOF states cannot converge. SOF states from multiconfigurational treatment are better choice for calculation of spin orbit interaction. ${ }^{71,73}$

The zero field splitting parameters of all the complexes have been investigated applying state-average complete active space self-consistent field (SA-CASSCF) calculation. The dynamical correlations are included using the $\mathrm{N}$-electron valence state perturbation theory (NEVPT2) method.

\subsection{Selection of active space and number of roots}

The proper choice of active space and nroots is crucial to achieve appropriate convergence in the multi-configurational methods. The minimal active space i.e. CAS $(5,5)$ containing five d-electrons in five d-orbitals of Fe does not predict the correct ground-spin state of 
the complex 2. Chowdhury et al. noticed that the spin state of complex is sensitive to axial Fe-P bond and suggested to include the $p_{z}$-orbitals of axial $\mathrm{P}$ ligands in the active space. ${ }^{49}$ Hence, the expanded active space i.e. CAS $(9,7)$ which includes the four $p_{z}$-electrons of $\mathrm{P}$-atom along with the five d-electrons of Fe in two $p_{z}$-orbitals of $\mathrm{P}$ and five d-orbitals of Fe is used for all the calculations. The active orbitals are shown in Figure 2a.

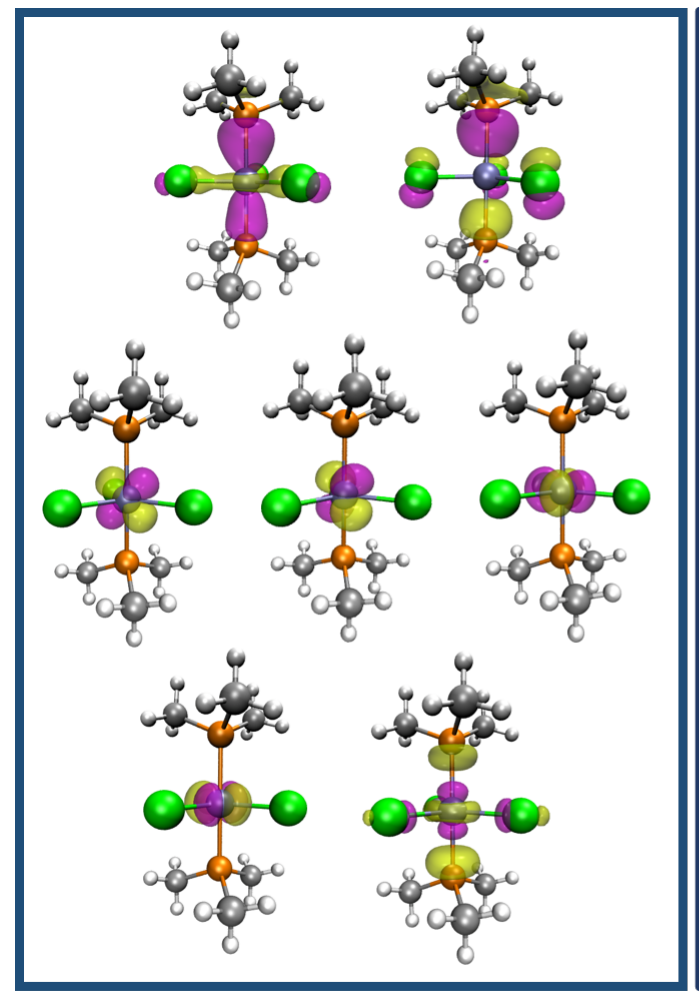

a.

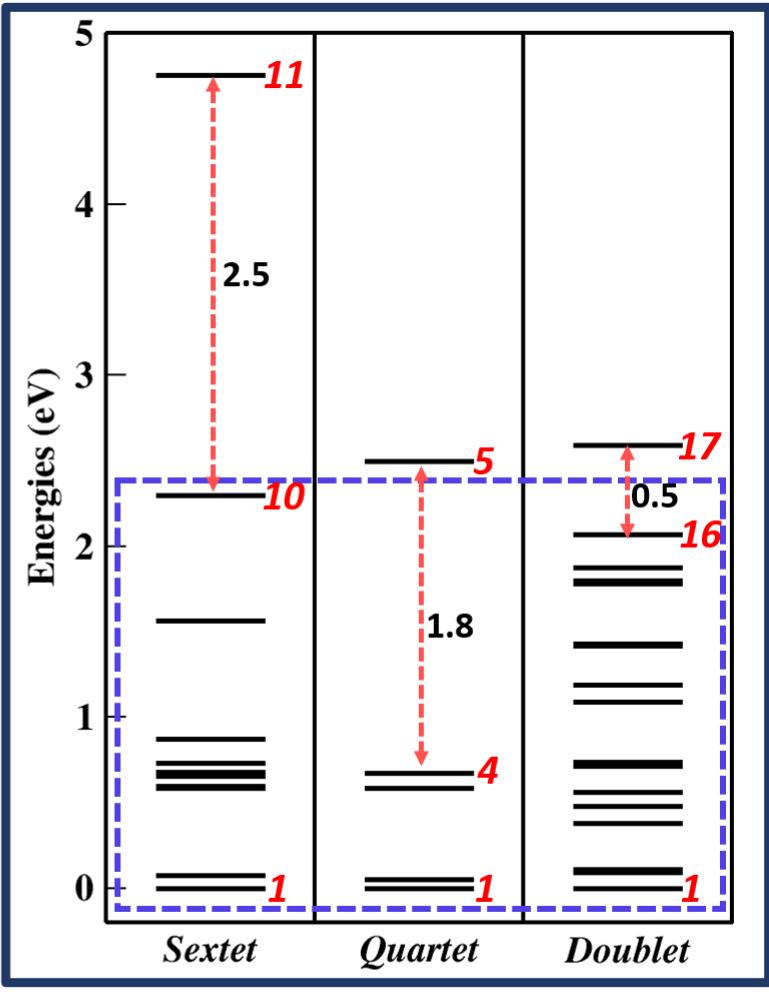

b.

Figure 2: (a.) Active orbitals generated after SA-CASSCF calculations with active space CAS $(9,7)$. (b.) Energy spectra of nroots of multiplets obtained from converged SA$\operatorname{CASSCF}(9,7)$ calculation with 21 roots of sextet, 224 roots of quartet and 490 roots of doublet.

The effect of inclusion of nroots of excited state and their contribution to magnetic anisotropy is important and is well explained by Llanos et al. ${ }^{74}$ The choice of nroots of particular multiplicity is important for reducing the computational cost and to include only those low lying excited states (roots), which actively participate in mixing of states. Also, including states lying higher in energy affects the description of optimized orbitals of lower lying states which are important. ${ }^{71,75}$ The selection of nroots is system specific 
and for the complexes under study, the procedure followed for the inclusion of appropriate nroots of particular multiplicity is thoroughly explained. It is observed that by the inclusion of all the roots of respective multiplicities, the calculated $D$ value is -18.25 $\mathrm{cm}^{-1}$ (for details, see Table S1 in supporting information) from SA-CASSCF calculations which is very small in comparison to $-50 \mathrm{~cm}^{-1}$ reported experimentally ${ }^{48}$ and NEVPT2 calculations did not converge due to computational demand of the calculation. It marks the significance of inclusion of only low lying excited roots of different multiplicities. The selection of nroots is based on the energy spectra of nroots of multiplets of complex 2 which is obtained from the converged $\operatorname{SA} \operatorname{CASSCF}(9,7)$ calculation with 21 roots of sextet, 224 roots of quartet and 490 roots of doublet (Figure $2 b$ ). It can be observed from the energy spectra that 10 roots of sextet, 4 roots of quartet and 16 roots of doublet lies within the range of $\sim 2 \mathrm{eV}$. Further, for sextet state, the energy gap between $10^{\text {th }}$ and $11^{\text {th }}$ number of root is quite large. Therefore, only 10 roots of sextet out of total 21 roots are considered for further calculations. For quartet state, although the $5^{\text {th }}$ root is close to $2 \mathrm{eV}$, but due to large energy gap between $4^{\text {th }}$ and $5^{\text {th }}$ root, only 4 low-lying roots are considered. On similar basis, 16 roots of doublet are taken into consideration. Hence, 10 roots of sextet, 4 roots of quartet and 16 roots of doublet out of total 21, 224 and 490 roots of respective multiplicities are considered. All the states of a given multiplicity are equally weighed.

\section{Results and Discussions}

A detailed investigation of the electronic structure and magnetic properties of all the complexes is done. In the following subsections, firstly the discussion of ground-spin state of all the complexes is presented. Followed by a comprehensive discussion, the spin-state energetics and the observation of spin-crossover in some complexes will be discussed. The origin of large zero field splitting is addressed in the subsequent subsection. 


\subsection{Ground-Spin State}

The complex 2 is optimized in the three possible spin states, i.e. HS ( $\mathrm{S}=5 / 2)$, IS ( $\mathrm{S}=3 / 2)$ and LS $(S=1 / 2)$ state. The geometry optimizations and the corresponding energy minima at the high-, low- and intermediate-spin states are confirmed by the absence of any imaginary vibrational frequencies. From the DFT optimized geometries, the IS quartet state is found to be the ground-spin state with the adiabatic energy difference of 20.40 $\mathrm{kJ} / \mathrm{mol}$ with respect to HS sextet state and $70.97 \mathrm{~kJ} / \mathrm{mol}$ compared to LS doublet state. The IS ground state for the complex is also observed by Feng et al. ${ }^{48}$ from DC susceptibility measurements data and Chowdhury et al. ${ }^{49}$ from $a b$ initio calculations respectively. All the other modelled complexes are optimized employing the same B3LYP-D3/def2TZVPP method. The DFT optimized energies of all the complexes (1-15) in all spin states (HS, IS and LS) are tabulated in Table S2 (Supporting Information). From the comparison of energies of complexes in HS, IS and LS state, it is observed that for all the complexes, the LS state is always higher in energy. The IS state is found to be the ground-spin state for complexes $2,3,4,11,12,13$ and 14 . Further, the prediction of ground-spin state for these complexes from DFT is also validated with SA-CASSCF/NEVPT2 calculations.

All the multireference calculations are performed using the CAS $(9,7)$ active space. The vertical excitation energies are calculated at the ground-spin state geometry of the respective complexes obtained from DFT. In agreement with DFT calculations for complex 2, 3, $4,11,12,13$ and 14, IS state is stabilized as ground state. Along with these, complex 10 and 15, are also observed to be in IS ground state for which the HS state is predicted to be the ground state from DFT. To understand the dissimilarity for these two complexes, the energy difference between the HS and IS state is investigated and is tabulated in Table 1. 
Table 1: Adiabatic energy difference $\left(\Delta \mathrm{E}_{H S-I S}^{\text {adia. }}\right)(\mathrm{kJ} / \mathrm{mol})$ between the HS and the IS state from DFT obtained at B3LYP-D3/def2-TZVPP level and the vertical excitation energies $\left(\triangle \mathrm{E}_{H S-I S}^{\text {vert. }}\right)(\mathrm{kJ} / \mathrm{mol})$ between the ground quartet and excited sextet states from SACASSCF $(9,7) / N E V P T 2$. The vertical excitation energies are calculated at the ground-spin state geometry of the respective complexes obtained from DFT. Energy difference is positive when the complex is stabilized in IS state and negative for HS stabilized complex.

\begin{tabular}{ccccc}
\hline Complex & \multirow{2}{*}{ Acronym } & $\Delta \mathbf{E}_{H S-I S}^{\text {adia. }}$ & \multicolumn{2}{c}{$\Delta \mathbf{E}_{H S}^{\text {vert. }}$ IS } \\
\cline { 3 - 5 } & & DFT & SA-CASSCF & SA-NEVPT2 \\
\hline $\mathbf{1}$ & $1_{H S}-\mathrm{PF}$ & -37.3 & -132.4 & -135.5 \\
$\mathbf{2}$ & $2_{I S}-\mathrm{PCl}$ & 20.4 & 53.9 & 12.9 \\
$\mathbf{3}$ & $3_{I S}-\mathrm{PBr}$ & 27.1 & 66.6 & 25.9 \\
$\mathbf{4}$ & $4_{I S}-\mathrm{PI}$ & 33.2 & 132.2 & 85.1 \\
$\mathbf{5}$ & $5_{H S}-\mathrm{NF}$ & -64.3 & -203.1 & -156.9 \\
$\mathbf{6}$ & $6_{H S}-\mathrm{NCl}$ & -56.2 & -69.6 & -139.1 \\
$\mathbf{7}$ & $7_{H S}-\mathrm{NBr}$ & -58.4 & -103.3 & -127.2 \\
$\mathbf{8}$ & $8_{H S}-\mathrm{NI}$ & -62.9 & -24.1 & -106.2 \\
$\mathbf{9}$ & $9_{H S}-\mathrm{AsF}$ & -54.2 & -66.4 & -183.2 \\
$\mathbf{1 0}$ & $10_{I S}-\mathrm{AsCl}$ & $-0.6(9.1)$ & 53.6 & 04.1 \\
$\mathbf{1 1}$ & $11_{I S}-\mathrm{AsBr}$ & 12.2 & 66.2 & 22.3 \\
$\mathbf{1 2}$ & $12_{I S}-\mathrm{AsI}$ & 21.8 & 77.6 & 40.1 \\
$\mathbf{1 3}$ & $13_{I S}-\mathrm{NPBr}$ & $0.6(13.4)$ & 66.4 & 14.9 \\
$\mathbf{1 4}$ & $14_{I S}-\mathrm{AsPBr}$ & 20.1 & 65.3 & 23.1 \\
$\mathbf{1 5}^{b}$ & $15_{I S}-\mathrm{NAsBr}$ & $-4.8(8.7)$ & 70.7 & 19.5 \\
\hline
\end{tabular}

${ }^{a}$ The values in bracket are calculated with reparametrized B3LYP with $15 \%$ HF exchange ${ }^{b}$ For this complex, $10 \%$ HF exchange is considered.

From the energy difference data, it is realized that the adiabatic energy difference between the HS and IS state from DFT is very small for complex 10 and 15. A small energy gap is also observed for complex 13. It has already been reported that hybrid functionals tend to stablise the HS state as the ground state when the energy difference between the two states is not too large. Therefore, for complexes with small energy difference i.e. 10, 13 and 15, we performed calculations with different hybrid and gradient corrected functionals like OPBE, PBE0, PBE and BLYP with the same basis set. To this end, the hybrid functionals i.e. OPBE and PBE0 are found to be biased towards the HS state whereas the gradient corrected functional i.e. PBE predicted the IS spin state as ground state. From BLYP functional, IS is observed to be ground state for complex 10 whereas HS for complex 13 and 15 (Table S3). It has been proposed that reparametrization of hybrid functionals 
by balanced admixture of exact and local exchange is necessary to produce the correct spin-state energetics. ${ }^{51,76}$ Therefore, we also reparametrized the hybrid B3LYP functional by varying the percentage of exact exchange admixture from 0 to $25 \%$ (originally its $20 \%$ for B3LYP) for complexes 10,13 and 15. It has been found that decreasing the percentage of exact exchange leads to correct ground-spin state as predicted by multireference calculations. A linear relationship is observed in $\Delta \mathrm{E}_{H S-I S}^{\text {adia. }}$ and percentage of exact exchange admixture (Figure 3) as found by Reiher et al. ${ }^{51,76,77}$

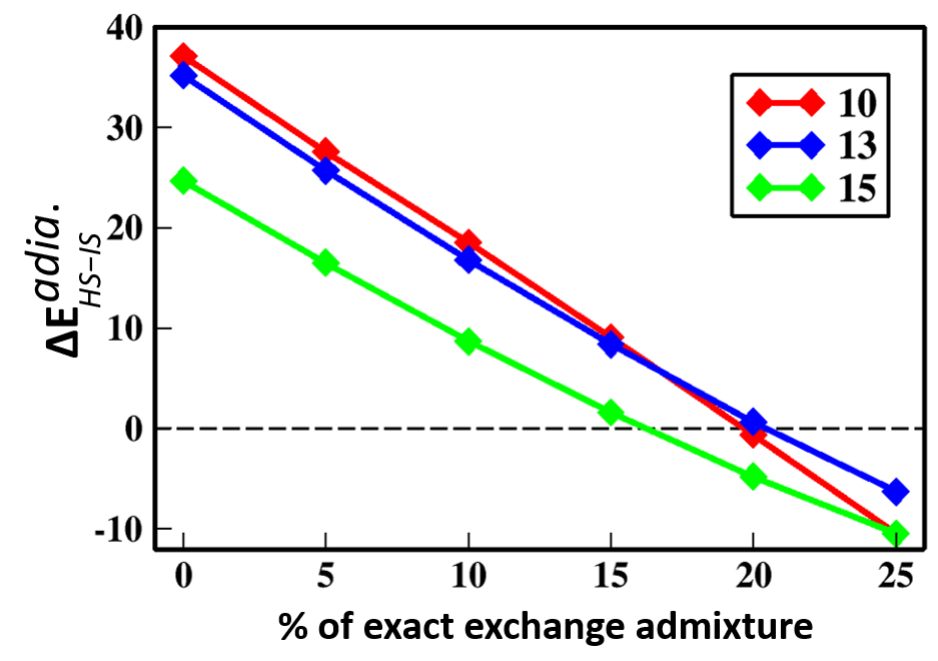

Figure 3: Variation of $\triangle \mathrm{E}_{H S-I S}^{\text {adia. }}$ with the $\%$ of exact exchange admixture in the hybrid functional B3LYP for complex 10,13 and 15. Originally there is 20\% exact exchange admixture in the B3LYP functional.

For the rest of the complexes, good correspondence is noticed in adiabatic energy difference from DFT and the vertical excitation energies from multireference methods. For complexes $1,5,6,7,8$ and $\mathbf{9}$, HS state is stabilized as the ground state with both DFT and multireference calculations.

\subsection{Spin-Crossover}

The spin-crossover (SCO) appears in systems with small energy difference in the spinstates. SCO in molecules is driven by the increase in entropy due to increase in the metalligand (M-L) bond lengths accompanying the transition from IS to HS state. In the com- 
plexes under study, the major structural changes associated with the change in spin state are the M-L bond lengths along the symmetry axis i.e. Fe-P for experimentally synthesized complex 2. The elongation of Fe-P bond lengths from 2.33 to $2.55 \AA$ i.e. a change of $0.22 \AA$ is obtained while switching from IS to HS state. The increase of M- $\mathrm{L}_{\text {axial }}$ bond lengths in HS state is due to population of electrons in the $\mathrm{d}_{z}^{2}$ orbital of central metal which is directed towards the axial metal-ligand bonds. Therefore, the ligands pointing towards this direction experience more coulomb repulsion in HS state. In all the complexes, the change in $\mathrm{M}-\mathrm{L}_{\text {axial }}$ bond length is observed in the range of $0.20-0.30 \AA$. However, a minor decrease of $0.01 \AA$ is observed for the $\mathrm{M}-\mathrm{L}_{\text {equatorial }}$ bond lengths for complex 2. For all the complexes, the change in equatorial bond lengths is not much pronounced (Table S5). The entropy is observed to increase for all the complexes while switching from IS to HS state due to increase in M-L bond lengths along axial direction. It suggests that SCO phenomenon is dominated by M-L bond lengths in axial direction only.

Table 2: Adiabatic energy difference $\left(\Delta \mathrm{E}_{H S-I S}^{\text {adia. }}\right)$, Free energy change $\left(\Delta \mathrm{G}_{H S-I S}^{\text {adia. }}\right)$, enthalpy change $\left(\Delta \mathrm{H}_{H S-I S}^{\text {adia. }}\right)$ and entropy change $\left(\mathrm{T} \Delta \mathrm{S}_{\mathrm{HS}-I S}^{\text {adia. }}\right)$ in $\mathrm{kJ} / \mathrm{mol}$ for all the complexes obtained at B3LYP-D3/def2-TZVPP level. $\Delta \mathrm{G}_{H S-I S}^{\text {adia. }}, \Delta \mathrm{H}_{H S-I S}^{\text {adia. }}$ and $\mathrm{T} \Delta \mathrm{S}_{H S-I S}^{\text {adia. }}$ are calculated at $300 \mathrm{~K}$.

\begin{tabular}{cccccc}
\hline Complex & Acronym & $\Delta \mathrm{E}_{H S-I S}^{\text {adia. }}$ & $\Delta \mathbf{G}_{H S-I S}^{\text {adia. }}$ & $\Delta \mathbf{H}_{H S-I S}^{\text {adia. }}$ & $\mathbf{T} \Delta \mathbf{S}_{H S-I S}^{\text {adia. }}$ \\
\hline $\mathbf{1}$ & $1_{H S}-\mathrm{PF}$ & -37.3 & -43.1 & -38.3 & 4.7 \\
$\mathbf{2}$ & $2_{I S}-\mathrm{PCl}$ & 20.4 & 11.9 & 18.9 & 7.1 \\
$\mathbf{3}$ & $3_{I S}-\mathrm{PBr}$ & 27.1 & 20.8 & 25.5 & 4.9 \\
$\mathbf{4}$ & $4_{I S}-\mathrm{PI}$ & 33.2 & 24.6 & 34.1 & 9.1 \\
$\mathbf{5}$ & $5_{H S}-\mathrm{NF}$ & -64.3 & -72.1 & -66.2 & 5.5 \\
$\mathbf{6}$ & $6_{H S}-\mathrm{NCl}$ & -56.2 & -64.5 & -58.6 & 6.1 \\
$\mathbf{7}$ & $7_{H S}-\mathrm{NBr}$ & -58.4 & -66.1 & -60.6 & 5.2 \\
$\mathbf{8}$ & $8_{H S}-\mathrm{NI}$ & -62.9 & -70.4 & -65.4 & 4.9 \\
$\mathbf{9}$ & $9_{H S}-\mathrm{AsF}$ & -54.2 & -58.6 & -57.2 & 1.3 \\
$\mathbf{1 0}$ & $10_{I S}-\mathrm{AsCl}$ & 9.1 & 0.5 & 7.5 & 7.0 \\
$\mathbf{1 1}$ & $11_{I S}-\mathrm{AsBr}$ & 12.3 & 4.6 & 10.7 & 5.7 \\
$\mathbf{1 2}$ & $12_{I S}-\mathrm{AsI}$ & 21.9 & 14.7 & 19.7 & 4.9 \\
$\mathbf{1 3}^{a}$ & $13_{I S}-\mathrm{NPBr}$ & 8.4 & 1.4 & 6.3 & 4.9 \\
$\mathbf{1 4}$ & $14_{I S}-\mathrm{AsPBr}$ & 20.1 & 10.8 & 21.1 & 10.2 \\
$\mathbf{1 5}^{b}$ & $15_{I S}-\mathrm{NAsBr}$ & 8.7 & 0.7 & 6.3 & 5.8 \\
\hline
\end{tabular}

${ }^{a}$ The values for these are calculated with reparametrized B3LYP with $15 \%$ HF exchange

${ }^{b}$ For this complex, $10 \%$ HF exchange is considered. 
Adiabatic energy difference from DFT provides an indication of SCO phenomenon. For the observation of spin-crossover, the energy gap between the two spin-states (i.e. $\Delta \mathrm{E}_{\text {HS-IS }}^{\text {adia. }}$ ) was suggested to be 0 to $25 \mathrm{~kJ} / \mathrm{mol}$ by Neese et al. ${ }^{78}$ However, the more rational approach is to compare the Gibbs free energy difference i.e. $\Delta \mathrm{G}_{H S-I S}^{\text {adia. }}$, which takes into account thermic and entropic corrections as well. The small value of Gibbs free energy difference between the spin-state for some complexes indicates the possibility for the observation of spin-crossover between them (Table 2). From the table, it is observed that $\Delta \mathrm{G}_{\mathrm{HS}-\mathrm{IS}}^{\text {adia. }}$ is small ( $<\sim 10 \mathrm{~kJ} / \mathrm{mol}$ ) for complexes $\mathbf{1 0}, \mathbf{1 1}, \mathbf{1 3}, \mathbf{1 4}$ and $\mathbf{1 5}$, indicating switching between the bi-stable spin-states and hence these will behave as the superior spin-crossover materials. It is also observed that by varying the ligands at axial positions, with increasing softness of ligands, i.e. from $\mathrm{N}$ to As, $\Delta \mathrm{G}_{H S-I S}^{\text {adia. }}$ decreases. However, by varying the ligands at equatorial positon i.e. from $\mathrm{F}$ to $\mathrm{I}, \Delta \mathrm{G}_{H S-I S}^{\text {adia. }}$ increases.

\subsection{Zero Field Splitting}

For the emergence of magnetic anisotropy in a molecule, both the sign and the magnitude of zero field splitting (ZFS) parameters are important. The large uniaxial anisotropy is signified by the negative sign of $D$ and its large magnitude together with small $E$, which influences the possibility of observing slow magnetic relaxation.

For complex 2, ZFS parameter, D, calculated by SA-CASSCF $(9,7)$ calculation for intermediate ground-spin state geometry is $-37.27 \mathrm{~cm}^{-1}$ (Table S1) which further improves to $-44.78 \mathrm{~cm}^{-1}$ (Table 3) upon inclusion of dynamical correlations using NEVPT2 calculation. The value is in nice agreement with the experimentally reported value of $-50 \mathrm{~cm}^{-1}$. From these NEVPT2 results, it is manifested that dynamical correlations play an important role in the calculation of ZFS value. Therefore, for all the modelled systems, $D$ value is reported by the inclusion of dynamical correlations. The reason for the observed high value of $D$ for complex 2 is the presence of axial symmetry which leads to quenching of Jahn-Teller distortion. ${ }^{49}$ This is also reflected in very small degeneracy breaking of lowest 
lying $d_{x z}$ and $d_{y z}$ orbitals (Figure 4). With such a small splitting of d-orbital in systems with close to ideal axial symmetry, ground and first excited states lie very close to each other in quasi-degenerate manner and leads to effective mixing and high first order spinorbit coupling. This is reflected in the ZFS value contribution of first excited state which accounts for the major part of total ZFS.

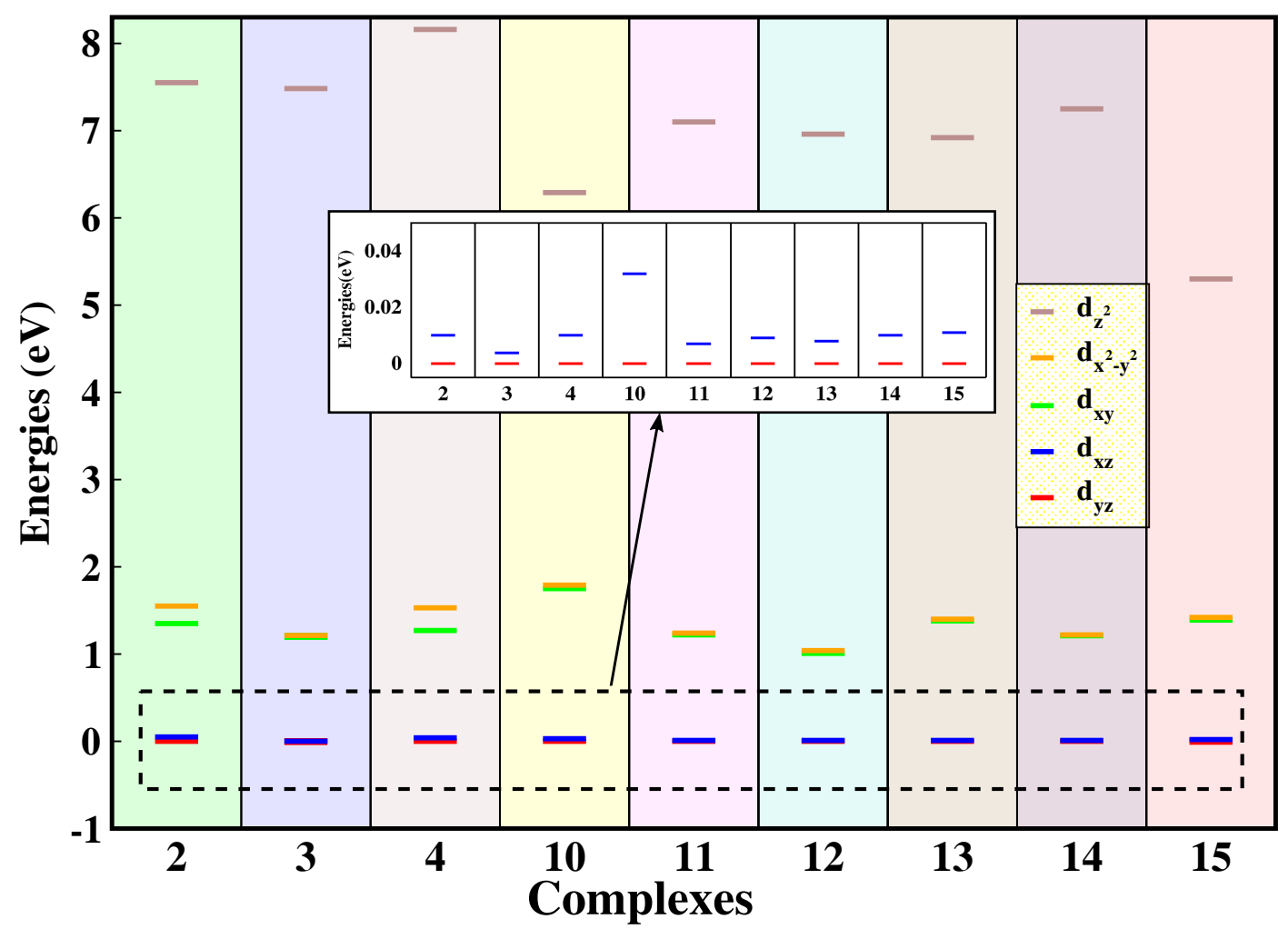

Figure 4: The $\mathrm{m}_{l}$-resolved 3d-orbital energy levels obtained in the SA-CASSCF $(9,7)$ calculations for all the 9 predicted SMMs. The ground state $3 \mathrm{~d}$-orbitals occupations for these complexes are $d_{y z}^{2} d_{x z}^{1} d_{x y}^{1} d_{x^{2}-y^{2}}^{1} d_{z^{2}}^{0}$. The computed large $D$-values for all these SMMs are primarily because of the first-order contribution to $\mathrm{D}$ due to mixing of ground state with the low-lying 1 st excited state i.e. $d_{y z}^{1} d_{x z}^{2} d_{x y}^{1} d_{x^{2}-y^{2}}^{1} d_{z^{2}}^{0}$ for all these complexes. The inset plot is the zoomed view of the energy difference between $\mathrm{d}_{y z}$ and $\mathrm{d}_{x z}$ orbitals. The smaller the gap between the later orbitals, larger the $D$-values.

Following the results for complex 2, the zero field splitting is calculated from NEVPT2 calculations on the wavefunction generated by SA-CASSCF for all the complexes in HS as well as IS state on their corresponding geometries. For complexes 2, 3, 4, 10, 11, 12, 13, 14 and 15 , the large negative ZFS in the range of -40 to $-60 \mathrm{~cm}^{-1}$ is calculated in the IS state 
Table 3: D value $\left(\mathrm{cm}^{-1}\right)$ of all complexes from SA-NEVPT2 calculations on top of CASSCF $(9,7)$ in HS and IS state on their respective geometries.

\begin{tabular}{cccc}
\hline Complex & Acronym & \multicolumn{2}{c}{ D value $\mathbf{( c m}^{-1}$ ) } \\
\cline { 3 - 4 } & & HS & IS \\
\hline $\mathbf{1}$ & $1_{H S}-\mathrm{PF}$ & -01.29 & -03.66 \\
$\mathbf{2}$ & $2_{I S}-\mathrm{PCl}$ & -01.56 & -44.78 \\
$\mathbf{3}$ & $3_{I S}-\mathrm{PBr}$ & -02.43 & -58.09 \\
$\mathbf{4}$ & $4_{I S}-\mathrm{PI}$ & -09.78 & -42.06 \\
$\mathbf{5}$ & $5_{H S}-\mathrm{NF}$ & -01.82 & -02.94 \\
$\mathbf{6}$ & $6_{H S}-\mathrm{NCl}$ & -00.84 & -02.21 \\
$\mathbf{7}$ & $7_{H S}-\mathrm{NBr}$ & -01.45 & -02.13 \\
$\mathbf{8}$ & $8_{H S}-\mathrm{NI}$ & -00.45 & -02.29 \\
$\mathbf{9}$ & $9_{H S}-\mathrm{AsF}$ & -00.31 & -01.72 \\
$\mathbf{1 0}$ & $10_{I S}-\mathrm{AsCl}$ & -01.30 & -51.66 \\
$\mathbf{1 1}$ & $11_{I S}-\mathrm{AsBr}$ & -01.80 & -54.89 \\
$\mathbf{1 2}$ & $12_{I S}-\mathrm{AsI}$ & -18.02 & -54.94 \\
$\mathbf{1 3}$ & $13_{I S}-\mathrm{NPBr}$ & -02.06 & -57.42 \\
$\mathbf{1 4}$ & $14_{I S}-\mathrm{AsPBr}$ & -02.10 & -54.86 \\
$\mathbf{1 5}$ & $15_{I S}-\mathrm{NAsBr}$ & -01.67 & -51.87 \\
\hline
\end{tabular}

on the respective optimized geometry. The value reduces to a relatively smaller magnitude, $<-3 \mathrm{~cm}^{-1}$ for the HS state on the geometry optimized in HS state. An exception is observed for complex 4 and $\mathbf{1 2}$ where a notable spin-orbit coupling is coming from heavy iodide ligand. ${ }^{79}$ The large SOC in these complexes lowers the energy of quartet excited state and brings it closer to the sextet ground state and gives a $D$ value of -9.78 and -18.02 $\mathrm{cm}^{-1}$ respectively. However, for other complexes which are stabilized in HS state, very small amount of zero field splitting, $<-4 \mathrm{~cm}^{-1}$, is observed in both the spin-states on their respective geometries. The reason for the lower value of ZFS in case of HS state is the quenching of orbital angular momentum in $\mathrm{Fe}(\mathrm{III})$ ion with $\mathrm{d}^{5}$ electronic configuration with the five singly occupied d-orbitals in the HS state. In case of an isotropic electronic distribution as in Fe(III) HS state, $D$ value should ideally be zero. ${ }^{80}$ This is due to the fact that first order spin-orbit coupling term goes to zero. The only contribution to ZFS comes from second-order spin-orbit coupling. Hence, the complexes with large ZFS in the IS state are studied in more details and are discussed in the following discussion. 
Table 4: Lowest spin-free energy levels $\left(\mathrm{cm}^{-1}\right)$ of the complexes for quartet excited states along with their individual contribution to $D$ and $E\left(\mathrm{~cm}^{-1}\right)$ computed using SACASSCF/NEVPT2 method with $(9,7)$ active space. Here, the wavefunction of the excited states 1, 2 and 3 presents the major contribution from $d_{y z}^{1} d_{x z}^{2} d_{x y}^{1} d_{x^{2}-y^{2}}^{1} d_{z^{2}}^{0}$ [12110], $d_{y z}^{1} d_{x z}^{1} d_{x y}^{2} d_{x^{2}-y^{2}}^{1} d_{z^{2}}^{0}$ [11210] and $d_{y z}^{1} d_{x z}^{1} d_{x y}^{1} d_{x^{2}-y^{2}}^{2} d_{z^{2}}^{0}$ [11120] configurations respectively. The CI coefficients of each excited state are given in the brackets.

\section{Complex Excited states Spin-free states Contb. D Contb. E}

\begin{tabular}{|c|c|c|c|c|}
\hline & $1(0.87)$ & 389.8 & -40.68 & -0.465 \\
\hline \multirow[t]{2}{*}{2} & $2(0.87)$ & 6465.0 & 01.80 & 1.830 \\
\hline & $3(0.87)$ & 7568.4 & 01.20 & -1.196 \\
\hline & $1(0.84)$ & 126.4 & -60.95 & -0.491 \\
\hline \multirow[t]{2}{*}{3} & $2(0.64)$ & 5603.8 & 01.72 & 1.497 \\
\hline & $3(0.64)$ & 5663.5 & 01.66 & -1.396 \\
\hline \multirow{3}{*}{4} & $1(0.87)$ & 215.0 & -48.79 & 0.116 \\
\hline & $2(0.87)$ & 5903.2 & 01.78 & -1.830 \\
\hline & $3(0.87)$ & 7711.7 & 01.12 & 1.108 \\
\hline \multirow{3}{*}{10} & $1(0.84)$ & 424.7 & -44.13 & -1.474 \\
\hline & $2(0.83)$ & 6269.3 & 01.82 & 0.965 \\
\hline & $3(0.83)$ & 7460.3 & 01.30 & -0.348 \\
\hline \multirow{3}{*}{11} & $1(0.81)$ & 162.2 & -57.38 & -0.418 \\
\hline & $2(0.83)$ & 5473.0 & 01.79 & 1.535 \\
\hline & $3(0.82)$ & 5594.6 & 01.67 & -1.380 \\
\hline \multirow{3}{*}{12} & $1(0.70)$ & 123.7 & -60.58 & -0.310 \\
\hline & $2(0.80)$ & 4187.9 & 02.23 & -2.210 \\
\hline & $3(0.80)$ & 4414.3 & 02.24 & 2.193 \\
\hline \multirow{3}{*}{13} & $1(0.52)$ & 107.0 & -57.53 & -0.812 \\
\hline & $2(0.77)$ & 4697.2 & 01.25 & -1.495 \\
\hline & $3(0.77)$ & 4802.4 & 01.82 & 1.643 \\
\hline \multirow{3}{*}{14} & $1(0.82)$ & 164.9 & -57.63 & -0.437 \\
\hline & $2(0.74)$ & 5506.3 & 01.65 & -1.508 \\
\hline & $3(0.74)$ & 5627.6 & 01.80 & 1.626 \\
\hline \multirow{3}{*}{15} & $1(0.70)$ & 123.7 & -53.13 & 0.224 \\
\hline & $2(0.72)$ & 4670.2 & 00.91 & -1.098 \\
\hline & $3(0.72)$ & 4727.5 & 01.86 & 1.098 \\
\hline
\end{tabular}

The ZFS can be correlated with the energy difference between the quartet ground and excited spin-free states. It has been well recognized that the excitation of electron between the same $\left|\mathrm{m}_{l}\right|$ states leads to negative contribution to $D$ whereas between the different $\left|\mathrm{m}_{l}\right|$ states leads to positive contribution to the overall value of zero field splitting. ${ }^{81}$ 
In CASSCF converged CI ground state wavefunction, major contribution comes from $d_{y z}^{2} d_{x z}^{1} d_{x y}^{1} d_{x^{2}-y^{2}}^{1} d_{z^{2}}^{0}$ configuration and that of first excited state from $d_{y z}^{1} d_{x z}^{2} d_{x y}^{1} d_{x^{2}-y^{2}}^{1} d_{z^{2}}^{0}$ configuration following the excitation of electron from $d_{y z}$ to $d_{x z}$. For second and third excited state, it comes from $d_{y z}^{1} d_{x z}^{1} d_{x y}^{2} d_{x^{2}-y^{2}}^{1} d_{z^{2}}^{0}$ and $d_{y z}^{1} d_{x z}^{1} d_{x y}^{1} d_{x^{2}-y^{2}}^{2} d_{z^{2}}^{0}$ configuration respectively. Since the excitation from $d_{y z}$ to $d_{x z}$ takes place between the same $\left|m_{l}\right|$ states, it will give negative contribution to $D$ whereas the excitation between $d_{y z}$ and $d_{x y}$ or $d_{x^{2}-y^{2}}$ leads to positive contribution due to transition between different $\left|\mathrm{m}_{l}\right|$ state (Table 4). Further, the magnitude of negative or positive contribution to $D$ depends on the energy difference between the ground and excited sub-levels. Since, there is a slight energy difference between the ground and first excited sub-levels in these complexes, therefore, the energy required for electronic transition from ground state to first excited state is quite low, leading to enhancement of spin-orbit coupling between the ground and the first excited state. It leads to large negative contribution to $D$ from the first excited state. However, due to larger energy gap between the ground state and second and third excited state, the magnitude of positive contribution is small compared to the larger negative contribution from first excited state, resulting in overall negative $D$ value for the complexes (Table 4).

Table 5: Average M-L $\mathrm{L}_{a x}$ and $\mathrm{M}-\mathrm{L}_{\text {equa. }}$. Mayer bond order and Löwdin atomic charges obtained at B3LYP-D3/def2-TZVPP level in conjunction with def2/J auxiliary basis set. $\mathrm{L}_{a x}$. and $\mathrm{L}_{\text {equa. }}$ are axial and equatorial ligands respectively.

\begin{tabular}{|c|c|c|c|c|c|c|}
\hline \multirow[t]{2}{*}{ Complex } & \multirow[t]{2}{*}{ Acronym } & \multicolumn{2}{|c|}{ Mayer bond order } & \multicolumn{3}{|c|}{ Löwdin atomic charges } \\
\hline & & M-L $a x$. & M-L $\mathbf{L}_{\text {equa. }}$ & Fe & P/N/As & $\mathrm{Cl} / \mathrm{Br} / \mathrm{I}$ \\
\hline 2 & $2_{I S}-\mathrm{PCl}$ & 0.57 & 0.80 & -1.29 & 0.86 & 0.01 \\
\hline 3 & $3_{I S}-\mathrm{PBr}$ & 0.56 & 0.76 & -1.33 & 0.86 & 0.04 \\
\hline 4 & $4_{I S}-\mathrm{PI}$ & 0.59 & 0.79 & -2.20 & 0.83 & 0.39 \\
\hline 10 & $10_{I S}-\mathrm{AsCl}$ & 0.49 & 0.79 & -1.37 & 1.01 & 0.02 \\
\hline 11 & $11_{\text {IS }}-\mathrm{AsBr}$ & 0.48 & 0.76 & -1.42 & 1.01 & 0.03 \\
\hline 12 & $12_{I S}$-AsI & 0.51 & 0.79 & -1.50 & 1.01 & 0.08 \\
\hline 13 & $13_{I S}-\mathrm{NPBr}$ & $0.30(\mathrm{~N}) / 0.63(\mathrm{P})$ & 0.83 & -1.13 & $0.23(\mathrm{~N}) / 0.88(\mathrm{P})$ & 0.07 \\
\hline 14 & $14_{I S}-\mathrm{AsPBr}$ & $0.44(\mathrm{P}) / 0.64(\mathrm{As})$ & 0.83 & -1.38 & $1.01(\mathrm{As}) / 0.86(\mathrm{P})$ & 0.04 \\
\hline 15 & $15_{I S}-\mathrm{NAsBr}$ & $0.23(\mathrm{~N}) / 0.62(\mathrm{As})$ & 0.84 & -1.16 & $0.23(\mathrm{~N}) / 1.01(\mathrm{As})$ & 0.06 \\
\hline
\end{tabular}

In modelled complex 3, with bromide at equatorial position replacing chloride in com- 
plex 2, an increase in $D$ value is observed. It can be seen from Table 4 that the energy difference between the ground and first excited state decreases from complex 2 to 3 , i.e. from 389.8 to $126.4 \mathrm{~cm}^{-1}$ respectively. Hence, the electronic transition energy also decreases for complex 3, leading to larger $D$ value. From the calculated d-orbital splitting diagram also, it can be seen that the energy difference between $d_{y z}$ and $d_{x z}$ decreases from complex 2 to 3 , indicating greater contribution to $D$ for complex 3 (shown in inset of Figure 4). However, for complex 4 , the energy difference is larger than that for 3 leading to largest contribution to $D$ from first excited state in case of complex 3 .

The overall $D$ value for complexes 2, 3 and 4 is related to the covalent character of the Fe-halide bonds. This is reflected in the Mayer's bond order (Table 5) where bond order of $\mathrm{Fe}-\mathrm{Br}(0.76)$ is smaller and $\mathrm{Fe}-\mathrm{Cl}(0.80)$ and $\mathrm{Fe}-\mathrm{I}(0.79)$ have comparable bond order leading to largest $D$ value for complex 3 with $\mathrm{Br}$ as equatorial ligand and similar $D$ value for complex 2 and 4 with comparable bond order. The halogen ligands are known for their $\pi$-donation ability, which increases from $\mathrm{Cl}$ to $\mathrm{I}$ in the group. From the Löwdin charge analysis (Table 5), it is observed that the negative charge on Fe and positive charge on halide increases from $\mathrm{Cl}$ to I which is due to more charge transfer from halogen to ligand as we progress down the group. Hence, the $D$ value is expected to increase with the increase in $\pi$-donation strength. The trend is followed from $\mathrm{Cl}$ to $\mathrm{Br}$ where $D$ value is observed to be increased. However, an unexpected decrease is observed with I at equatorial position.

For complexes 10, 11 and 12, trimethylarsine are positioned at the axial orientation. It is well known that softness of atom increases while progressing down the group. It is expected that with heavier and softer ligand i.e. As in place of $\mathrm{P}$, the value of $D$ should increase. Therefore, the $D$ values for As containing ligands are larger than that of $\mathrm{P}$ containing ligands. From the analysis of Mayer bond order (Table 5), it is observed that the bond order of Fe-As (0.40-0.51) is smaller than Fe-P (0.51-0.59) bond order, accounting for the reason of more $D$ value for As containing ligands than the P containing ligands. 
Also, with soft ligand i.e. As, there will be more charge transfer from axial ligand to metal, which is observed from the Löwdin atomic charges where negative and positive charge on Fe and As increases as compared to that with P ligand, indicating easy transfer of electrons from ligand to metal in case of As. Hence, increase in $D$ value is observed with trimethylarsine ligands. The increase in $D$ value in complexes 10, 11 and 12 with replacement of halide ligands can be elucidated from the increased charge transfer while progressing from $\mathrm{Cl}$ to I at equatorial position. Less charge transfer from ligand to metal for complex 10 accounts for the smaller $D$ value. Complexes 11 and 12 have comparable charge transfer leading to almost equal $D$ values. Complexes 13, 14 and 15 with asymmetric ligands exhibit zero field splitting in the range from -52 to $-58 \mathrm{~cm}^{-1}$ in the IS ground state. The proportional energy difference between the quartet ground and first excited sub-level for the three complexes affirms the observed comparable $D$ value.

Almost negligible rhombicity, $|E / D|<0.03$ is calculated for these complexes which points towards high axiality of the magnetic anisotropy and suppression of relaxation through quantum tunneling (Table S9).

From the above discussion, complexes 10, 11, 13, 14 and 15 possess Gibbs free energy difference below $10 \mathrm{~kJ} / \mathrm{mol}$ and in parallel, possess large zero field splitting in the IS state and reduces to small value in the HS state. Hence, these complexes emerge to be possible candidates for both spin-crossover and molecular magnetic materials.

\section{Conclusions}

We have modelled fourteen Fe(III)-TBP based complexes, out of which nine are stabilized in the IS ground state. They exhibit high magnetic anisotropy with $D$ values in the range -40 to $-60 \mathrm{~cm}^{-1}$ due to quasi-degenerate ground and first excited states. The ZFS is well correlated with the energy difference between the quartet ground and excited sub-levels. The ZFS is increased by varying the ligands from $\mathrm{N}$ to As at axial position. Among lig- 
ands at equatorial position, highest $D$ value in the series is observed with the bromide ligand followed by iodide and chloride. The complexes containing fluoride at equatorial position possess HS ground state and hence, are not a good choice as ligands for SMM materials. Parallel to this, complexes with $\mathrm{N}$ at axial position also possess $\mathrm{HS}$ ground state. However, the complexes containing P or As in combination with $\mathrm{N}$ ligands provide superior materials. These asymmetric ligands point towards a new avenue in modelling of single ion magnets giving a way to design and synthesize SMMs based on asymmetric ligand substitution.

For the observation of spin-crossover phenomenon, adiabatic energy difference between the two spin states and more relevant Gibbs free energy difference is a decisive parameter. It is observed that $\Delta \mathrm{G}_{H S-I S}^{\text {adia. }}$ decreases with increasing softness of ligands at axial position i.e. from $\mathrm{N}$ to As whereas it increases when the ligands are substituted from $F$ to I at equatorial position. Complexes 10, 11, 13, 14 and 15 among all the designed complexes posses small $\left|\Delta \mathrm{G}_{H S-I S}^{\text {adia. }}\right|$ (i.e. $<\sim 10.0 \mathrm{~kJ} / \mathrm{mol}$ ) indicating the spin-crossover behavior of these systems.

\section{Acknowledgement}

The authors thank Prof. Sabyashachi Mishra for various helpful discussion. R.K. thanks CSIR, India for the JRF fellowship with grant number 09/1129(0016)/2019-EMR-I. Financial support from Department of Science and Technology through SERB-ECR project No. ECR/2016/000362 and SERB-CRG project No. CRG/2019/003237 are highly acknowledged.

Supporting Information Available: Benchmarking of nroots, Energetics comparison between different spin-states, SS- and SA-CASSCF results, optimized structural parameters, DFT and SA-CASSCF/NEVPT2 calculated ZFS parameters. This material is available free of charge via the Internet at http:/ / pubs.acs.org. 


\section{References}

(1) Cavallini, M.; Gomez-Segura, J.; Ruiz-Molina, D.; Massi, M.; Albonetti, C.; Rovira, C.; Veciana, J.; Biscarini, F. Magnetic information storage on polymers by using patterned single-molecule magnets. Angew. Chem. 1994, 117, 910-914.

(2) Affronte, M.; Troiani, F.; Ghirri, A.; Candini, A.; Evangelisti, M.; radini, V.; Carretta, S.; Santini, P.; Amoretti, G.; Tuna, F.; Timco, G.; Winpenny, R. E. P. Single molecule magnets for quantum computation. J. Phys. D: Appl. Phys. 2007, 40, 2999.

(3) Bogani, L.; Wernsdorfer, W. Molecular spintronics using single-molecule magnets. Nat. Mater. 2008, 7, 179-186.

(4) Dreiser, J.; Wackerlin, C.; Ali, M. E.; Piamonteze, C.; Donati, F.; Singha, A.; Pedersen, K. S.; Rusponi, S.; Bendix, J.; Oppeneer, P. M., et al. Exchange interaction of strongly anisotropic tripodal erbium single-ion magnets with metallic surfaces. ACS Nano 2014, 8, 4662-4671.

(5) Corradini, V.; Ghirri, A.; Candini, A.; Biagi, R.; del Pennino, U.; Dotti, G.; Otero, E.; Choueikani, F.; Blagg, R. J.; McInnes, E. J. L.; Affronte, M. Magnetic cooling at a single molecule level: a spectroscopic investigation of isolated molecules on a surface. Adv. Mater. 2013, 25, 2816-2820.

(6) Gatteschi, D.; Sessoli, R.; Villain, J. Molecular Nanomagnets; 2006; Vol. 5.

(7) Frost, J. M.; Harriman, K. L. M.; Murugesu, M. The rise of 3-d single-ion magnets in molecular magnetism: towards materials from molecules? Chem. Sci. 2016, 7, 24702491.

(8) Craig, G. A.; Murrie, M. 3d single-ion magnets. Chem. Soc. Rev. 2015, 44, 2135-2147.

(9) Bar, A. K.; Pichon, C.; Sutter, J.-P. Magnetic anisotropy in two- to eight-coordinated 
transition-metal complexes: Recent developments in molecular magnetism. Coord. Chem. Rev. 2016, 308, 346-380.

(10) Cahier, B.; Perfetti, M.; Zakhia, G.; Naoufal, D.; El-Khatib, F.; Guillot, R.; Rivière, E.; Sessoli, R.; Barra, A.-L.; Guihéry, N.; Mallah, T. Magnetic anisotropy in pentacoordinate $\mathrm{Ni}^{I I}$ and $\mathrm{Co}^{I I}$ complexes: Unraveling electronic and geometrical contributions. Chem. Euro. J. 2017, 23, 3648-3657.

(11) Ding, Y.-S.; Chilton, N. F.; Winpenny, R. E. P.; Zheng, Y.-Z. On approaching the limit of molecular magnetic anisotropy: A near-perfect pentagonal bipyramidal dysprosium(III) single-molecule magnet. Angew. Chem., Int. Ed. 2016, 55, 16071-16074.

(12) Randall McClain, K.; Gould, C. A.; Chakarawet, K.; Teat, S. J.; Groshens, T. J.; Long, J. R.; Harvey, B. G. High-temperature magnetic blocking and magnetostructural correlations in a series of dysprosium(III) metallocenium single-molecule magnets. Chem. Sci. 2018, 9, 8492-8503.

(13) Guo, F.-S.; Day, B. M.; Chen, Y.-C.; Tong, M.-L.; Mansikkamäki, A.; Layfield, R. A. Magnetic hysteresis up to 80 kelvin in a dysprosium metallocene single-molecule magnet. Science 2018, 362, 1400-1403.

(14) Gupta, S. K.; Shanmugan, S.; Rajeshkumar, T.; Borah, A.; Damjanović, M.; Schulze, M.; Wernsdorfer, W.; Rajaraman, G.; Murugavel, R. A single-ion singleelectron cerrous magnet. Dalton Trans. 2019, 48, 15928-15935.

(15) Gupta, S. K.; Murugavel, R. Enriching lanthanide single-ion magnetism through symmetry and axiality. Chem. Commun. 2018, 54, 3685-3696.

(16) Kalita, P.; Acharya, J.; Chandrasekhar, V. Mononuclear pentagonal bipyramidal Ln (III) complexes: Syntheses and magnetic properties. J. Magn. Magn. Mater 2020, 498, 166098. 
(17) Singh, S. K.; Cramer, C. J.; Gagliardi, L. Correlating electronic structure and magnetic anisotropy in actinide complexes $\left[\mathrm{An}(\mathrm{COT})_{2}\right], \mathrm{An}(\mathrm{III} / \mathrm{IV})=\mathrm{U}, \mathrm{Np}$, and Pu. Inorg. Chem. 2020, 59, 6815-6825.

(18) Gaggioli, C. A.; Gagliardi, L. Theoretical investigation of plutonium-based singlemolecule magnets. Inorg. Chem. 2018, 57, 8098-8105.

(19) Liu, J.-L.; Chen, Y.-C.; Tong, M.-L. Symmetry strategies for high performance lanthanide-based single-molecule magnets. Chem. Soc. Rev. 2018, 47, 2431-2453.

(20) Harman, W. H.; Harris, T. D.; Freedman, D. E.; Fong, H.; Chang, A.; Rinehart, J. D.; Ozarowski, A.; Sougrati, M. T.; Grandjean, F.; Long, G. J.; Long, J. R.; Chang, C. J. Slow magnetic relaxation in a family of trigonal pyramidal iron(II) pyrrolide complexes. J. Am. Chem. Soc. 2010, 132, 18115-18126.

(21) Yao, X.-N.; Du, J.-Z.; Zhang, Y.-Q.; Leng, X.-B.; Yang, M.-W.; Jiang, S.-D.; Wang, Z.-X.; Ouyang, Z.-W.; Deng, L.; Wang, B.-W.; Gao, S. Two-coordinate Co(II) imido complexes as outstanding single-molecule magnets. J. Am. Chem. Soc. 2017, 139, 373-380.

(22) Zadrozny, J. M.; Xiao, D. J.; Atanasov, M.; Long, G. J.; Grandjean, F.; Neese, F.; Long, J. R. Magnetic blocking in a linear iron(I) complex. Nat. Chem. 2013, 5, 577581.

(23) Hołyńska, M.; Premužić, D.; Jeon, I.-R.; Wernsdorfer, W.; Clérac, R.; Dehnen, S. $\left[\mathrm{Mn}_{6}^{I I I} \mathrm{O}_{3} \mathrm{Ln}_{2}\right]$ Single-molecule magnets: Increasing the energy barrier above $100 \mathrm{~K}$. Euro. J. Chem. 2011, 17, 9605-9610.

(24) Dreiser, J.; Pedersen, K. S.; Piamonteze, C.; Rusponi, S.; Salman, Z.; Ali, M. E.; SchauMagnussen, M.; Thuesen, C. A.; Piligkos, S.; Weihe, H., et al. Direct observation of a ferri-to-ferromagnetic transition in a fluoride-bridged $3 \mathrm{~d}-4 \mathrm{f}$ molecular cluster. Chem. Sci. 2012, 3, 1024-1032. 
(25) Gütlich, P.; Garcia, Y.; Goodwin, H. A. Spin crossover phenomena in Fe(II) complexes. Chem. Soc. Rev. 2000, 29, 419-427.

(26) Marchivie, M.; Guionneau, P.; Howard, J. A. K.; Chastanet, G.; Létard, J.-F.; Goeta, A. E.; Chasseau, D. Structural characterization of a photoinduced molecular switch. J. Am. Chem. Soc. 2002, 124, 194-195.

(27) Bousseksou, A.; Molnár, G.; Tuchagues, J.-P.; Menéndez, N.; Épiphane Codjovi,; Varret, F. Triggering the spin-crossover of $\mathrm{Fe}(\mathrm{phen})_{2}(\mathrm{NCS})_{2}$ by a pressure pulse. Pressure and magnetic field induce mirror effects. C R Chim 2003, 6, 329-335.

(28) Ali, M. E.; Staemmler, V.; Illas, F.; Oppeneer, P. M. Designing the redox-driven switching of ferro- to antiferromagnetic couplings in organic diradicals. J. Chem. Theory Comput. 2013, 9, 5216-5220.

(29) Rudavskyi, A.; Sousa, C.; de Graaf, C.; Havenith, R. W. A.; Broer, R. Computational approach to the study of thermal spin crossover phenomena. J. Chem. Phys. 2014, 140, 184318.

(30) Raymo, F. M. Digital processing and communication with molecular switches. Adv. Mater. 2002, 14, 401-414.

(31) Viciano Chumillas, M.; Blondin, G.; Krzystek, J.; Ozerov, M.; Armentano, D.; Schnegg, A.; Lohmiller, T.; Telser, J.; Lloret, F.; Cano, J. Single-ion magnetic behaviour in an iron(III) porphyrin complex: A dichotomy between high-spin and 5/2-3/2 spin admixture. Chem. Euro. J. 2020, DOI: 10.1002/chem.202003052.

(32) Stavretis, S. E.; Atanasov, M.; Podlesnyak, A. A.; Hunter, S. C.; Neese, F.; Xue, Z.-L. Magnetic transitions in iron porphyrin halides by inelastic neutron scattering and ab initio studies of zero-field splittings. Inorg. Chem. 2015, 54, 9790-9801. 
(33) Yergeshbayeva, S.; Hrudka, J. J.; Lengyel, J.; Erkasov, R.; Stoian, S. A.; DragulescuAndrasi, A.; Shatruk, M. Heteroleptic Fe(II) complexes with $\mathrm{N}_{4} \mathrm{~S}_{2}$ coordination as a platform for designing spin-crossover materials. Inorg. Chem. 2017, 56, 11096-11103.

(34) Arroyave, A.; Lennartson, A.; Dragulescu-Andrasi, A.; Pedersen, K. S.; Piligkos, S.; Stoian, S. A.; Greer, S. M.; Pak, C.; Hietsoi, O.; Phan, H.; Hill, S.; McKenzie, C. J.; Shatruk, M. Spin crossover in Fe(II) complexes with $\mathrm{N}_{4} \mathrm{~S}_{2}$ coordination. Inorg. Chem. 2016, 55, 5904-5913.

(35) Feng, M.; Tong, M.-L. Single ion magnets from 3d to 5f: Developments and strategies. Chem. Euro. J. 2018, 24, 7574-7594.

(36) Rajaraman, G.; Sarkar, A.; Dey, S. Role of coordination number and geometry in controlling the magnetic anisotropy in Fe (II), Co (II) and Ni (II) single-ion magnets. Chem. Eur. J. 2020, DOI:10.1002/chem.202003211.

(37) Singh, S. K.; Rajaraman, G. Deciphering the origin of giant magnetic anisotropy and fast quantum tunnelling in Rhenium(IV) single-molecule magnets. Nat. Commun. 2016, 7, 10669.

(38) Gruden-Pavlović, M.; Perić, M.; Zlatar, M.; García-Fernández, P. Theoretical study of the magnetic anisotropy and magnetic tunnelling in mononuclear $\mathrm{Ni}(\mathrm{II})$ complexes with potential molecular magnet behavior. Chem. Sci. 2014, 5, 1453-1462.

(39) Marriott, K. E. R.; Bhaskaran, L.; Wilson, C.; Medarde, M.; Ochsenbein, S. T.; Hill, S.; Murrie, M. Pushing the limits of magnetic anisotropy in trigonal bipyramidal Ni(II). Chem. Sci. 2015, 6, 6823-6828.

(40) Craig, G. A.; Sarkar, A.; Woodall, C. H.; Hay, M. A.; Marriott, K. E. R.; Kamenev, K. V.; Moggach, S. A.; Brechin, E. K.; Parsons, S.; Rajaraman, G.; Murrie, M. Probing the origin of the giant magnetic anisotropy in trigonal bipyramidal $\mathrm{Ni}(\mathrm{II})$ under high pressure. Chem. Sci. 2018, 9, 1551-1559. 
(41) Mossin, S.; Tran, B. L.; Adhikari, D.; Pink, M.; Heinemann, F. W.; Sutter, J.; Szilagyi, R. K.; Meyer, K.; Mindiola, D. J. A mononuclear Fe(III) single molecule magnet with a 3/2-5/2 spin crossover. J. Am. Chem. Soc. 2012, 134, 13651-13661.

(42) Ruamps, R.; Batchelor, L. J.; Guillot, R.; Zakhia, G.; Barra, A.-L.; Wernsdorfer, W.; Guihéry, N.; Mallah, T. Ising-type magnetic anisotropy and single molecule magnet behaviour in mononuclear trigonal bipyramidal Co(II) complexes. Chem. Sci. 2014, 5, 3418-3424.

(43) Hay, M. A.; McMonagle, C. J.; Wilson, C.; Probert, M. R.; Murrie, M. Trigonal to pentagonal bipyramidal coordination switching in a $\mathrm{Co}(\mathrm{II})$ single-ion magnet. Inorg. Chem. 2019, 58, 9691-9697.

(44) Hay, M. A.; Sarkar, A.; Marriott, K. E. R.; Wilson, C.; Rajaraman, G.; Murrie, M. Investigation of the magnetic anisotropy in a series of trigonal bipyramidal Mn(II) complexes. Dalton Trans. 2019, 48, 15480-15486.

(45) Saber, M. R.; Dunbar, K. R. Ligands effects on the magnetic anisotropy of tetrahedral cobalt complexes. Chem. Commun. 2014, 50, 12266-12269.

(46) Goswami, T.; Misra, A. Ligand effects toward the modulation of magnetic anisotropy and design of magnetic systems with desired anisotropy characteristics. J. Phys. Chem. A 2012, 116, 5207-5215.

(47) Drahoš, B.; Herchel, R.; Trávníček, Z. Impact of halogenido coligands on magnetic anisotropy in seven-coordinate Co(II) complexes. Inorg. Chem. 2017, 56, 5076-5088.

(48) Feng, X.; Hwang, S. J.; Liu, J.-L.; Chen, Y.-C.; Tong, M.-L.; Nocera, D. G. Slow magnetic relaxation in intermediate spin $\mathrm{S}=3 / 2$ mononuclear Fe(III) complexes. J. Am. Chem. Soc. 2017, 139, 16474-16477. 
(49) Roy Chowdhury, S.; Mishra, S. Ab initio investigation of magnetic anisotropy in intermediate spin iron (III) complexes. J. Chem. Phys. 2018, 149, 234302.

(50) Swart, M. Accurate spin-state energies for iron complexes. J. Chem. Theory Comput. 2008, 4, 2057-2066.

(51) Reiher, M.; Salomon, O.; Hess, B. A. Reparameterization of hybrid functionals based on energy differences of states of different multiplicity. Theor. Chem. Acc. 2001, 107, 48-55.

(52) Radoń, M. Benchmarking quantum chemistry methods for spin-state energetics of iron complexes against quantitative experimental data. Phys. Chem. Chem. Phys. 2019, $21,4854-4870$.

(53) Singh, S. K.; Gupta, T.; Badkur, P.; Rajaraman, G. Magnetic anisotropy of mononuclear $\mathrm{Ni}(\mathrm{II})$ complexes: On the importance of structural diversity and the structural distortions. Chem. Eur. J 2014, 20, 10305-10313.

(54) Becke, A. D. A new mixing of Hartree-Fock and local density-functional theories. J. Chem. Phys. 1993, 98, 1372-1377.

(55) Lee, C.; Yang, W.; Parr, R. G. Development of the Colle-Salvetti correlation-energy formula into a functional of the electron density. Phys. Rev. B 1988, 37, 785-789.

(56) Weigend, F.; Ahlrichs, R. Balanced basis sets of split valence, triple zeta valence and quadruple zeta valence quality for $\mathrm{H}$ to $\mathrm{Rn}$ : Design and assessment of accuracy. Phys. Chem. Chem. Phys. 2005, 7, 3297-3305.

(57) Neese, F.; Wennmohs, F.; Hansen, A.; Becker, U. Efficient, approximate and parallel Hartree-Fock and hybrid DFT calculations. A 'chain-of-spheres' algorithm for the Hartree-Fock exchange. Chem. Phys. 2009, 98-109. 
(58) Neese, F. The ORCA program system. Wiley Interdiscip. Rev. Comput. Mol. Sci. 2012, $2,73-78$.

(59) Izsák, R.; Neese, F. An overlap fitted chain of spheres exchange method. J. Chem. Phys. 2011, 135, 144105.

(60) Weigend, F. Hartree Fock exchange fitting basis sets for H to Rn. J. Comput. Chem. 2007, 29, 167-175.

(61) Grimme, S.; Antony, J.; Ehrlich, S.; Krieg, H. A consistent and accurate ab initio parametrization of density functional dispersion correction (DFT-D) for the 94 elements H-Pu. J. Chem. Phys. 2010, 132, 154104.

(62) Grimme, S.; Ehrlich, S.; Goerigk, L. Effect of the damping function in dispersion corrected density functional theory. J. Comput. Chem. 2011, 32, 1456-1465.

(63) Åke Malmqvist, P.; Roos, B. O. The CASSCF state interaction method. Chem. Phys. Lett. 1989, 155, $189-194$.

(64) Angeli, C.; Cimiraglia, R.; Malrieu, J.-P. N-electron valence state perturbation theory: a fast implementation of the strongly contracted variant. Chem. Phys. Lett. 2001, 350, $297-305$.

(65) Angeli, C.; Cimiraglia, R.; Evangelisti, S.; Leininger, T.; Malrieu, J.-P. Introduction of n-electron valence states for multireference perturbation theory. J. Chem. Phys. 2001, $114,10252-10264$.

(66) Löwdin, P. On the non-orthogonality problem connected with the use of atomic wave functions in the theory of molecules and crystals. J. Chem. Phys. 1950, 18, 365-375.

(67) Mayer, I. Charge, bond order and valence in the ab initio SCF theory. Chem. Phys. Lett. 1983, 97, 270-274. 
(68) Helmich-Paris, B. CASSCF linear response calculations for large open-shell molecules. J. Chem. Phys. 2019, 150, 174121.

(69) Roemelt, M.; Krewald, V.; Pantazis, D. A. Exchange coupling interactions from the density matrix renormalization group and N-electron valence perturbation theory: Application to a biomimetic mixed-valence manganese complex. J. Chem. Theory Comput. 2018, 14, 166-179.

(70) Ganyushin, D.; Neese, F. First-principles calculations of zero-field splitting parameters. J. Chem. Phys. 2006, 125, 024103.

(71) Maurice, R.; Bastardis, R.; Graaf, C. d.; Suaud, N.; Mallah, T.; Guihéry, N. Universal theoretical approach to extract anisotropic spin hamiltonians. J. Chem. Theory Comput. 2009, 5, 2977-2984.

(72) Neese, F. Efficient and accurate approximations to the molecular spin-orbit coupling operator and their use in molecular g-tensor calculations. J. Chem. Phys. 2005, 122, 034107.

(73) Maurice, R. Zero-field anisotropic spin hamiltonians in first-row transition metal complexes: Theory, models and applications. Ph.D. thesis, 2011.

(74) Llanos, L.; Aravena, D. Effect of low spin excited states for magnetic anisotropy of transition metal mononuclear single molecule magnets. Inorganics 2018, 6, 24.

(75) Maurice, R.; Sivalingam, K.; Ganyushin, D.; Guihéry, N.; de Graaf, C.; Neese, F. Theoretical determination of the zero-field splitting in copper acetate monohydrate. Inorg. Chem. 2011, 50, 6229-6236.

(76) Reiher, M. Theoretical study of the Fe(phen $)_{2}(\mathrm{NCS})_{2}$ spin-crossover complex with reparametrized density functionals. Inorg. Chem. 2002, 41, 6928-6935. 
(77) Salomon, O.; Reiher, M.; Hess, B. A. Assertion and validation of the performance of the B3LYP* functional for the first transition metal row and the G2 test set. J. Chem. Phys. 2002, 117, 4729-4737.

(78) Ye, S.; Neese, F. Accurate modeling of spin-state energetics in spin-crossover systems with modern density functional theory. Inorg. Chem. 2010, 49, 772-774.

(79) Chowdhury, S. R.; Mishra, S. Heavy ligand atom induced large magnetic anisotropy in Mn (II) complexes. Phys. Chem. Chem. Phys. 2017, 19, 16914-16922.

(80) Neese, F.; Solomon, E. I. Calculation of zero-field splittings, g-values, and the relativistic nephelauxetic effect in transition metal complexes. Application to high-spin ferric complexes. Inorg. Chem. 1998, 37, 6568-6582.

(81) Gomez-Coca, S.; Cremades, E.; Aliaga-Alcalde, N.; Ruiz, E. Mononuclear singlemolecule magnets: Tailoring the magnetic anisotropy of first-row transition-metal complexes. J. Am. Chem. Soc. 2013, 135, 7010-7018. 


\section{Graphical TOC Entry}

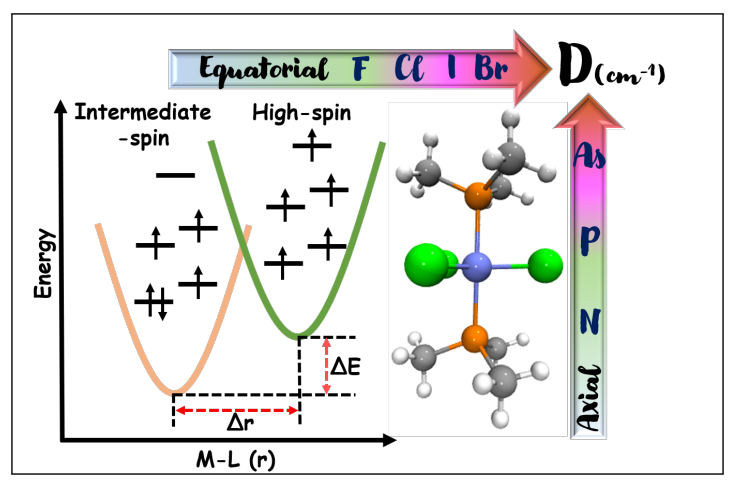

